\title{
Treatment plan optimization incorporating respiratory motion
}

\author{
Tiezhi Zhang, ${ }^{\text {a) }}$ Robert Jeraj, and Harry Keller \\ Department of Medical Physics, University of Wisconsin, Madison, Wisconsin 53706
}

Weiguo Lu and Gustavo H. Olivera

Tomotherapy, Inc., Madison, Wisconsin 53717

Todd R. McNutt

Philips Radiation Oncology Systems, Madison, Wisconsin 53719

Thomas R. Mackie and Bhudatt Paliwal

Department of Medical Physics, University of Wisconsin, Madison, Wisconsin 53706

and Department of Human Oncology, University of Wisconsin, Madison, Wisconsin 53792

(Received 30 October 2003; revised 24 March 2004; accepted for publication 25 March 2004; published 27 May 2004)

\begin{abstract}
Similar to conventional conformal radiotherapy, during lung tomotherapy, a motion margin has to be set for respiratory motion. Consequently, large volume of normal tissue is irradiated by intensive radiation. To solve this problem, we have developed a new motion mitigation method by incorporating target motion into treatment optimization. In this method, the delivery-breathing correlation is determined prior to treatment plan optimization. Beamlets are calculated by using the CT images at the corresponding breathing phases from a dynamic (four-dimensional) image sequence. With the displacement vector fields at different breathing phases, a set of deformed beamlets is obtained by mapping the dose to the primary phase. Optimization incorporating motion is then performed by using the deformed beamlets obtained by dose mapping. During treatment delivery, the same breathing-delivery correlation can be reproduced by instructing the patient to breathe following a visually displayed guiding cycle. This method was tested using a computer-simulated deformable phantom and a real lung case. Results show that treatment optimization incorporating motion achieved similar high dose conformality on a mobile target compared with static delivery. The residual motion effects due to imperfect breathing tracking were also analyzed. (C) 2004 American Association of Physicists in Medicine. [DOI: 10.1118/1.1739672]
\end{abstract}

\section{INTRODUCTION}

Helical tomotherapy ${ }^{1,2}$ is an intensity modulated radiotherapy (IMRT) delivery modality developed at the University of Wisconsin and TomoTherapy, Inc. Tomotherapy delivers intensity modulated beams from $360^{\circ}$ gantry angles in a helical pattern resulting in conformal radiation to the target. Fan shaped beam intensity is modulated by a binary multileaf collimator (MLC) that is able to open and close its leaves in less than $40 \mathrm{~ms}$. Tomotherapy delivery may be of advantage for lung cancer treatment. Lung cancer targets are usually surrounded by a dose limiting structure-the normal lung tissue. Conventional delivery causes high entrance and exit doses whereas tomotherapy is able to spread the delivery to a larger volume. A previous treatment planning study showed that dose escalation by tomotherapy could be as high as $160 \mathrm{~Gy}$ in certain patients. ${ }^{3}$

Targets inside lung usually move significantly due to respiratory motion. A generous motion margin must be allowed and large volume of normal tissue is irradiated with intensive radiation. Measured with a real-time tumor/internal marker tracking system, the range of tumor motion was noted to be $5.5-10 \mathrm{~mm}$ in the lateral direction, $6.8-15.9 \mathrm{~mm}$ in the craniocaudal direction, and $8.1-14.6 \mathrm{~mm}$ in the ventrodorsal direction. ${ }^{4}$ The magnitude of motion depends on the location of the target (upper, middle, or lower lobe) within the lung and the details of the attachment of the tumor to other anatomical features.
Beside large motion margin, motion effects on IMRT delivery have been extensively studied during the past several years. Hot and cold spots in dose distribution were observed due to the interplay between the target and MLC leaf motion. ${ }^{5,6}$ Other studies also showed that non-uniformity can be improved after multifraction deliveries. ${ }^{7,8}$ For fractionated treatments, targets receive different amount dose in each fraction. As a result, different overall biological effects may be observed.

In order to maximize the benefits of tomotherapy to lung cancer treatments, a motion control or compensation method will enable additional sparing of normal tissue from intensive radiation as much as possible. Currently, motion control and compensation techniques can be classified as

(1) Free breathing gating,

(2) Breath-hold gating,

(3) Real time target tracking.

Free breathing gating ${ }^{9-11}$ sets a beam-on window to a specific portion of the patient's breathing cycle. Treatment is prolonged by three to four times depending on the duty cycle setting. Due to the rotational beam delivery, free breathing gating is more difficult to implement on tomotherapy than conventional delivery.

In breath-hold gating, ${ }^{12-14}$ patients hold their breath at deep inspiration and beam delivery happens during the breath-hold period. During deep inspiration, lung volume in- 


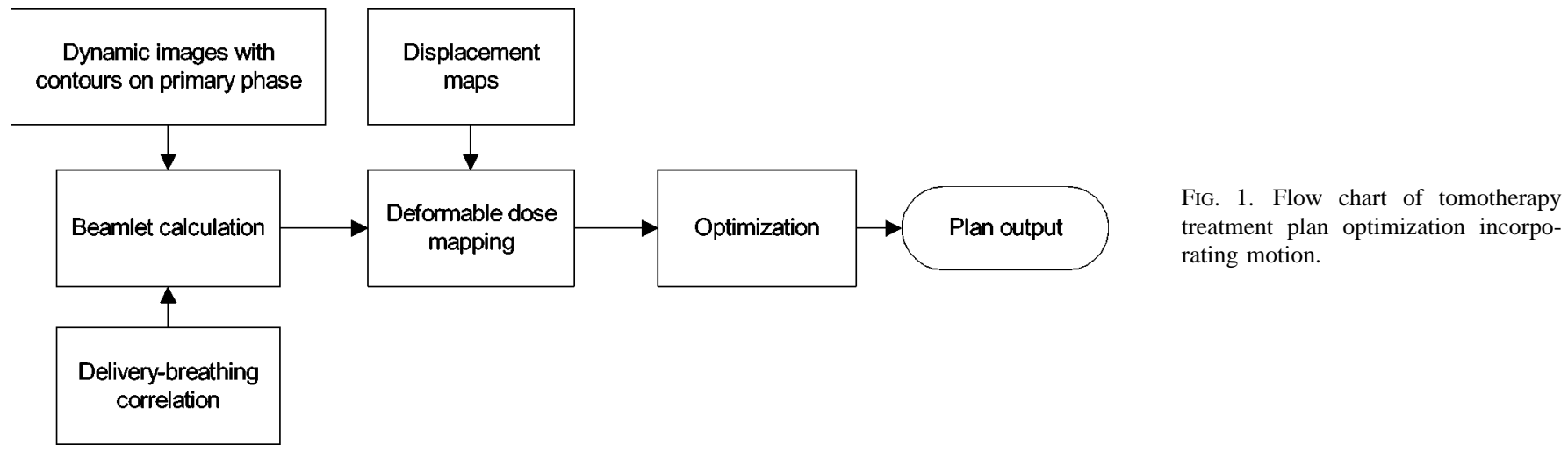

creases as much as 30\%. More normal tissue is thereby spared from radiation. The major drawbacks of this method are patient tolerance and treatment prolongation. Additional time has to be added to let the patient recover from breathholds. Only a portion of lung patients are able to hold their breath long enough to finish treatment with an acceptable time prolongation.

Four-dimensional (4D) radiotherapy by target tracking ${ }^{15,16}$ is a newly developed motion mitigation technique. In this technique, dynamic MLC leaf motions are adjusted to track the target motion. Continuous beam delivery does not prolong treatment time. However, due to the MLC mechanical limitation, usually only linear rigid body target motion is allowed. Tomotherapy delivers radiation helically, its binary MLC is not able to track superior-inferior motion which moves target in and out of the treatment plane. Although the independent jaw pair is technically capable of tracking the tumor in that direction, this method may impose more limitations on target motion and has not been developed at this time.

Of the current motion mitigation techniques, only the breath-hold method can be easily applied to tomotherapy at this time. A new technique with better patient tolerance is desired to make tomotherapy benefit the majority of lung patients. For this purpose, a new 4D tomotherapy treatment technique is developed. Contrary to previous techniques whose treatment planning is performed on static CT images, the new method uses 4D CT data set in beamlet dose calculation and includes the target motion into treatment optimization. This method, in principle, imposes no target motion limitation and preserves high dose conformality to mobile targets.

\section{MATERIALS AND METHODS}

\section{A. Theory}

During tomotherapy treatment the gantry rotates with a constant speed while the couch moves through the gantry. During the delivery, the patient is trained to control their breathing to follow a fixed, pre-determined pattern. Thus, the deformation state of the lung at any instance can be predicted if the treatment delivery is synchronized with the breathing cycle. The unique property of a continuous timeline of delivery with tomotherapy enables a new motion mitigation method by incorporating respiratory motion into treatment plan optimization. The procedure of motion incorporated treatment plan optimization for tomotherapy is shown in Fig. 1.

\section{Dynamic image acquisition}

With a still image and its displacement map at any instance, a dynamic (4D) image sequence can be obtained by using an image warping method. ${ }^{17,18}$ Due to the deformation, the pixel density of the image changes with time according to

$$
\rho_{0}(\vec{x})=\rho_{t}\left(\vec{x}+\vec{u}_{f}(\vec{x}, t)\right) \times J,
$$

where $\rho_{0}(\vec{x})$ is the density value at the position $\vec{x}$ of the original image, $\rho_{t}(\vec{x})$ is density value of the image at time $t$, $u_{f}$ is the forward displacement vector field and is a function of $x$ and $t, J$ is the Jacobian of the deformation gradient and defined by $J=\operatorname{det}\left(\delta_{i, j}+\partial u_{f}^{i} \partial x^{j}\right)$ where $i$ and $j$ are $x, y$, and $z$ directions, respectively.

Directly warping the image using Eq. (1a) causes overlapping or absence of values at some pixel positions. To avoid the defects, backward warping is used to create dynamic images at different phases:

$$
\rho_{t}(\vec{x})=\rho_{0}\left(\vec{x}+\vec{u}_{b}(\vec{x}, t)\right) \frac{1}{J},
$$

where $\vec{u}_{b}$ is the backward displacement vector field in a relation to $\vec{u}_{f}$ as $\vec{u}_{b}(\vec{x}, t)=-\vec{u}_{f}\left(\vec{x}+\vec{u}_{b}, t\right) . \vec{u}_{b}$ is obtained by interpolation since the equation is unsolvable.

\section{Delivery-breathing correlation}

Tomotherapy gantry typically rotates with a constant speed of about $20 \mathrm{~s}$ per rotation. With voluntarily control from the patient, the breathing phase may also be predicted. The correlation between delivery and breathing can be set prior to treatment planning. This correlation should be preserved during the treatment. The correlation between delivery and the breathing cycle is shown schematically in Fig. 2.

\section{Beamlet dose calculation}

The formulation of superposition/convolution (S/C) algorithm for dose calculation on the static phantom is 


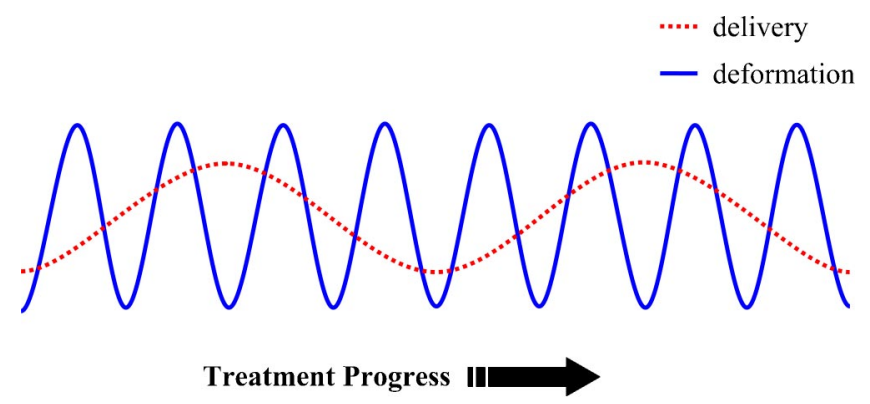

FIG. 2. Schematic diagram of the correlation between delivery phases (gantry angles) and deformation phases. The gantry rotation and target deformation have different but fixed periods. The correlation between delivery and phase is set prior to treatment planning and reproduced during the treatments.

$$
D(\vec{x})=\int d E \int d \vec{x}^{\prime}\left(\frac{\mu}{\rho_{x^{\prime}}}\right) E \psi\left(E, \rho_{\vec{x}^{\prime}}, \vec{x}^{\prime}\right) k\left(E, \vec{x}-\vec{x}^{\prime}\right),
$$

where $D(\vec{x})$ is the dose deposited at position $\vec{x}, E$ is the photon energy, $\vec{x}^{\prime}$ is the position of the primary photon interaction site, $\mu / \rho_{x}$ is mass attenuation coefficient, $\psi$ is the fluence of the beam, and $k$ is the kernel.

In the proposed method, the dynamic image sequence is used to determine the time dependent dose distribution for each beamlet. The formulation for $\mathrm{S} / \mathrm{C}$ calculation is adjusted as

$$
\begin{aligned}
D_{t}(\vec{x})= & \int d E \int d \vec{x}^{\prime}\left(\frac{\mu}{\rho\left(\vec{x}^{\prime}, t\right)}\right) E \psi\left(E, \rho\left(\vec{x}^{\prime}, t\right), \vec{x}^{\prime}\right) \\
& \times k\left(E, \vec{x}-\vec{x}^{\prime}\right),
\end{aligned}
$$

where $D_{t}(\vec{x})$ is the dose deposited at position $\vec{x}$ at instance $t$. The time dependent deformation is included in $\rho(\vec{x}, t)$ from the dynamic image sequence. During beamlet calculation, the image at the corresponding phase is chosen according to the breathing-phase correlation.

\section{Deformable dose mapping}

A primary phase is defined as the breathing phase for which regions of interest are contoured. It can be any phase of the dynamic image sequence. Deformable image registra-

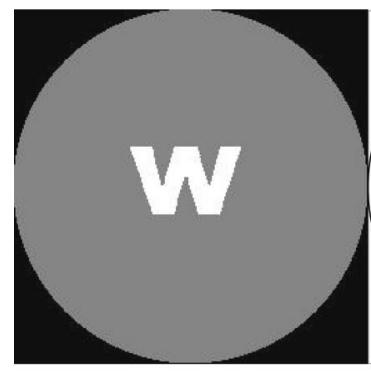

(a)

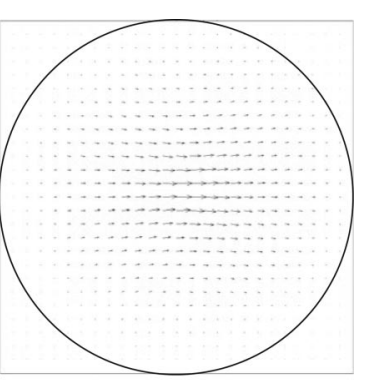

(b)
FIG. 3. A 2D deformable phantom. (a) A cylinder phantom with a "W" shape target inside. Both the normal tissue and the target have water equivalent density $1.0 \mathrm{~g} / \mathrm{cm}^{3}$. (b) The forward displacement vector field $\vec{\mu}_{f}$.

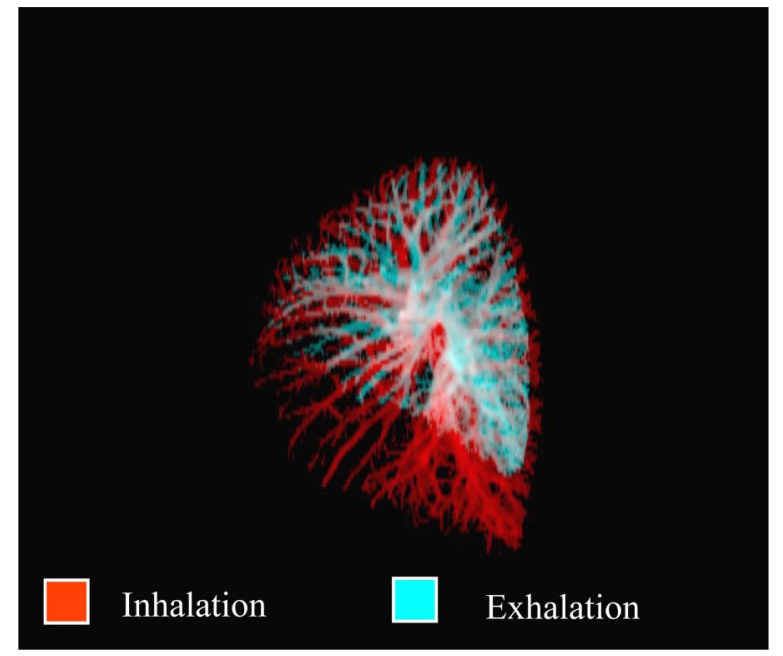

(a)

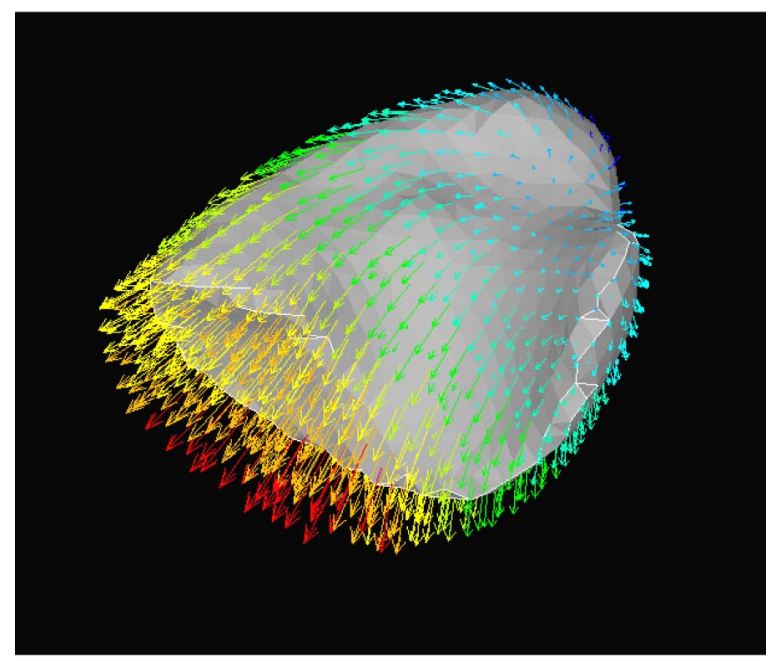

(b)

FIG. 4. (a) Lung images scanned at the inhalation and exhalation phases by breath holdings. (b) The displacement vector map between the two phases.

tion gives the displacement maps of the lung relative to the primary phase. The beamlets at each breathing phase are then mapped back to a primary phase as

$$
D_{0}(\vec{x})=D_{t}\left(\vec{x}+\vec{u}_{f}(\vec{x}, t)\right),
$$

where $D_{0}$ is the dose for the primary phase. The forward displacement vector field $\vec{u}_{f}$ is the same as that in Eq. (1a). The dose mapping is an inverse process of image warping but without the mass conservation factor $J$ in Eq. (1a). The density changes are included in the $\mathrm{S} / \mathrm{C}$ dose calculation by Eq. (2b).

\section{Treatment plan optimization}

Beamlet based optimization minimizes the residual defined as 

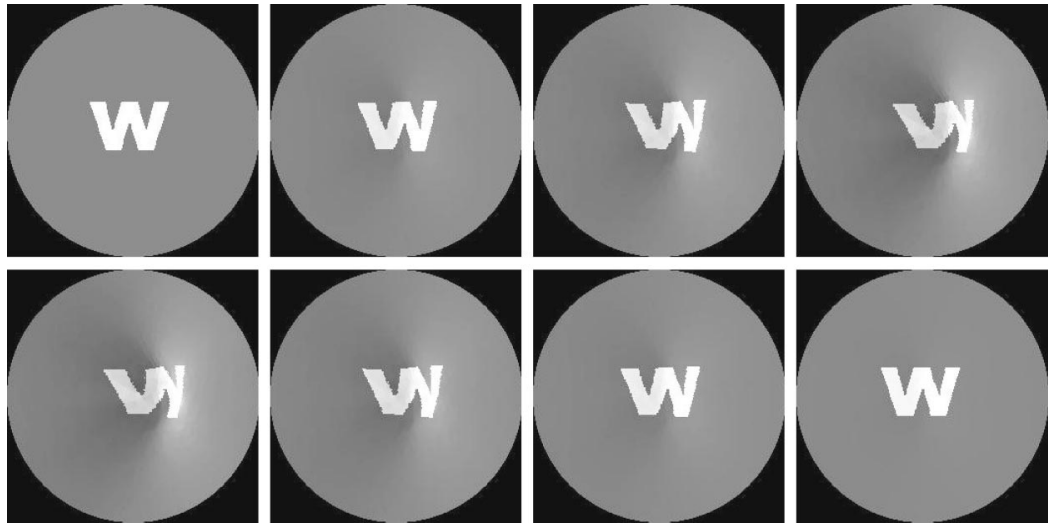

FIG. 5. A dynamic image sequence generated by mass conserved image warping. The images are separated with an interval of 0.625 second and the deformation cycle period is 5 seconds.

$$
\text { residual }=\int w(\vec{x})\left(D_{p}(\vec{x})-\sum_{i} \psi^{i} D^{i}(\vec{x})\right)^{2} d v,
$$

where $w(\vec{x})$ is the weighting factor, $D_{p}$ is prescribed dose, $D_{0}^{i}$ is the $i$ th beamlet dose per energy fluence distribution obtained from dose mapping, and $\psi^{i}$ is the fluence intensity.

In order to include the deformation information, the deformed beamlets $\left(D_{0}\right)$ obtained from dose mapping by Eq. (3) are used in optimization. The optimization algorithm is adjusted as

$$
\text { residual }=\int w(\vec{x})\left(D_{p}(\vec{x})-\sum_{i} \psi^{i} D_{t}^{i}\left(\vec{x}+\vec{u}_{f}(\vec{x}, t)\right)\right)^{2} d v .
$$

Motion is incorporated into the optimization by the term $\vec{u}_{f}(\vec{x}, t)$. The result of tomotherapy plan optimization is a set of beamlet intensities $\psi^{i}$ for each leaf position at different gantry angles.

\section{B. Two-dimensional phantom simulation}

The motion incorporated optimization was simulated by a computer generated deformable phantom. A cylindrical phantom image with a "W" shape target inside was created using MATLAB software (The MathWorks Inc., Natick, MA). The phantom had a water equivalent density of $1 \mathrm{~g} / \mathrm{cm}^{3}$ at a deformation-free state. Its diameter was $25.6 \mathrm{~cm}$. A displace-

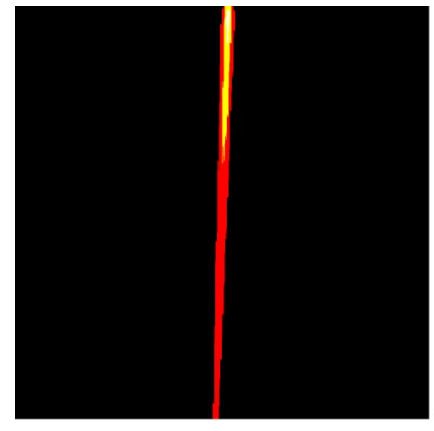

(a)

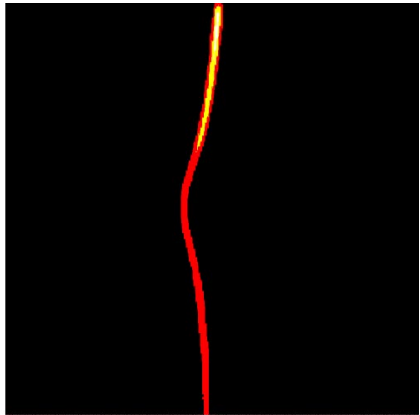

(b)
FIG. 6. Examples of beamlet dose distributions before (a) and after (b) dose mapping. The dose distribution was deformed due to phantom deformation. Total 51 projections distributed evenly from 360 degree gantry angles. ment vector map with the maximum displacement of about 3 $\mathrm{cm}$ in the center was arbitrarily created. A sinusoidal motion pattern was applied. Figure 3 shows the image of the phantom and its maximum displacement vector field.

Tomotherapy delivery was simulated by parallel beamlets rotating around the phantom. $6 \mathrm{MV}$ polyenergetic photon beams were used in the $\mathrm{S} / \mathrm{C}$ dose calculation. For each projection, a field of view (FOV) of $20 \mathrm{~cm}$ was covered by 50 beamlets with a width of $4 \mathrm{~mm}$ for each beamlet. (The beamlet width of an actual tomotherapy machine is $6.25 \mathrm{~mm}$.) A total of 51 projections were evenly distributed in a $360^{\circ}$ gantry angle. For dynamic delivery, the gantry was simulated to rotate with a period of $20 \mathrm{~s}$, the phantom deformed with a period of $5 \mathrm{~s}$, which is typical of breathing cycle period. There is no need for the relationship between the gantry rotation period and the breathing period to be an integer multiple. Beamlets were calculated to the phantom image sequence according to the delivery-breathing phase correlation. A total of 2550 beamlets were calculated for the twodimensional phantom case. The beamlets were then mapped back to the primary phase (deformation free phase) according to Eq. (3). In order to compare the results, another set of beamlets were calculated on the static phantom. A conventional simulated annealing algorithm was used for optimizations. The prescription was at least $95 \%$ dose to the target and $0 \%$ dose to the normal tissue. The weighting factor between the target and normal tissue was 100:1.

\section{Three-dimensional lung case study}

Lung images at two different breathing phases were obtained by breath holding CT scans. A finite element model based deformable image registration gave the displacement vector field of the lung between the two scanned phases. The results were shown in Fig. 4. This image registration method was described in detail in other publications. ${ }^{18,19}$ The displacement vector field for intermediate phases was obtained by linear interpolation.

During CT scans, the patient's breathing was monitored by a calibrated spirometry system (Medgraphics, St. Paul, $\mathrm{MN}) .{ }^{20}$ One of the cycles from the patient's normal breathing curve was then selected as the guiding cycle. A periodic and predictable breathing guide was then reconstructed by re- 


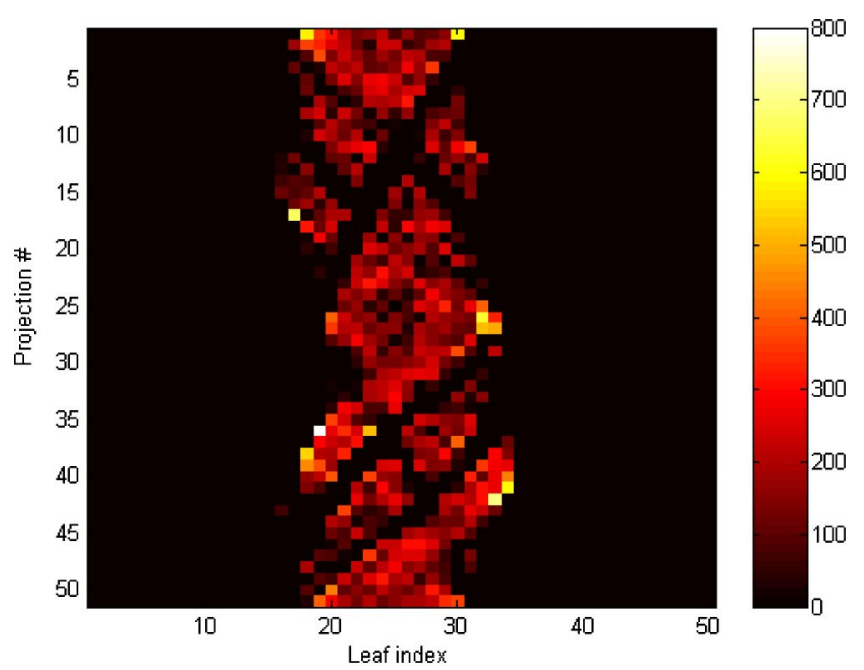

(a)

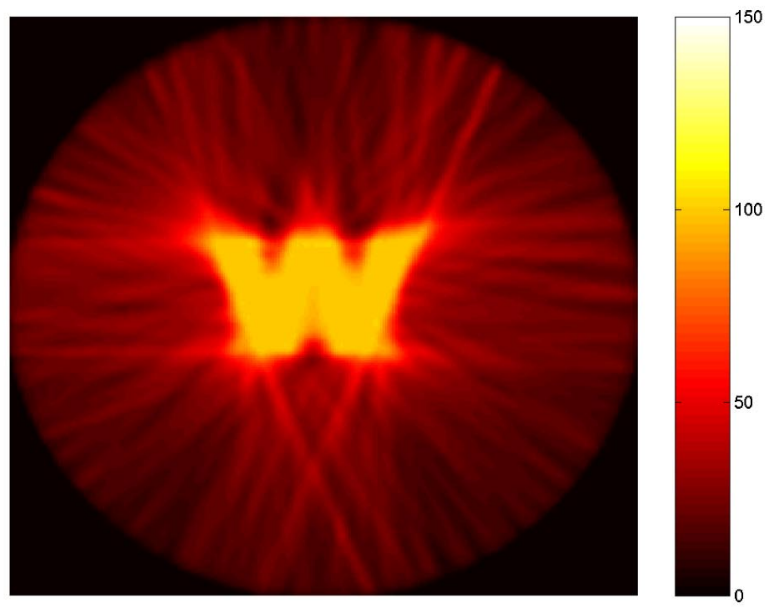

(b)

FIG. 7. Optimization results for a stationary target (a) the sinogram, (b) dose distribution. The dose is highly conformal $(\mathrm{DSC}=0.925)$ and uniform. The prescribed dose is $95 \%$ to the target.

peating the guiding cycle. The interpolated lung images were correlated with the breathing guide by calculating the lung volume changes. Assuming tomotherapy gantry rotates with a period of $20 \mathrm{~s}$, the correlation between the breathing guide and delivery phase (projection number) was then set.

With the displacement map at different breathing phases, a 4D lung image sequence was generated by image warping according to Eq. (1b). The inhalation phase was selected as the primary phase. Region of interest (ROI) contours were created on the primary phase using the treatment planning software Pinnacle ${ }^{\mathrm{TM}}$ (Philips Radiation Oncology System, Madison, WI). The contours of region of the ROI at other breathing phases were automatically generated with the knowledge of voxel displacements. The dynamic image sequence was then imported into the commercial tomotherapy optimizer (TomoTherapy, Inc., Middleton, WI). Five sets of beamlets were calculated for 10 breathing phases (5 inhala-

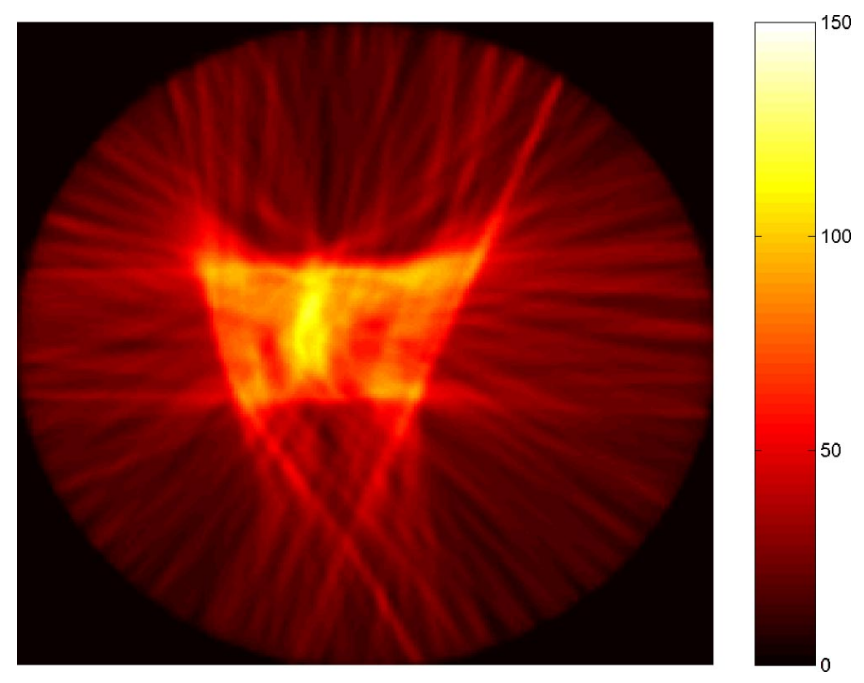

FIG. 8. The actual dose delivered on a deforming phantom if no motion mitigation is applied. Severe hot and cold spots are observed and the dose is not conformal to the "W" shape target $(\mathrm{DSC}=0.590)$.

tion, 5 exhalation). One set of beamlets for dynamic delivery was then picked out according to the preset deliverybreathing phase correlation. The beamlets were then mapped back to the primary phase according to Eq. (3). The same simulated annealing algorithm as the phantom study was used in optimization. The prescription was at least $95 \%$ dose to the target and $0 \%$ dose to the normal tissue. The weighting factor between target and normal tissue was 100:1.

\section{Dose calculation with tracking errors}

Due to patient's tracking error, the delivery-breathing correlation may not be perfectly reproduced. The actual dose delivered with human tracking error may be calculated as

$$
D_{f}(\vec{x})=\sum_{i} \psi^{i} D_{t}^{i}\left(\vec{x}+\vec{u}_{f}^{p}(\vec{x}, t)\right)
$$

where $D_{f}$ is final dose distribution, $\vec{u}_{f}^{p}$ is the actual deformation at time $t$ and is different from the forward displacement map $\vec{u}_{f}$ which is set by the guiding cycles. Equation (5a) gives the exact dose distribution for the delivery with tracking errors. However, using this equation to reconstruct dose requires the calculation of another set of beamlets which is a computationally expensive procedure for tomotherapy. However, with relatively small tracking errors, Eq. (5a) can be approximated as

$$
D_{f}(\vec{x})=\sum_{i} \psi^{i} D_{t}^{i}\left(\vec{x}+\vec{u}_{f}(\vec{x}, t)+\delta_{i} \times \vec{u}_{f}\left(\vec{x}, t_{\max }\right)\right),
$$

where $t_{\max }$ is the instance that the target undergoes the largest deformation and the dimensionless tracking errors $\delta_{i}$ are normally distributed with the mean of 0 and standard deviation of $\sigma$. The value of $\sigma$ is subject to change to simulate different degree of tracking errors. 


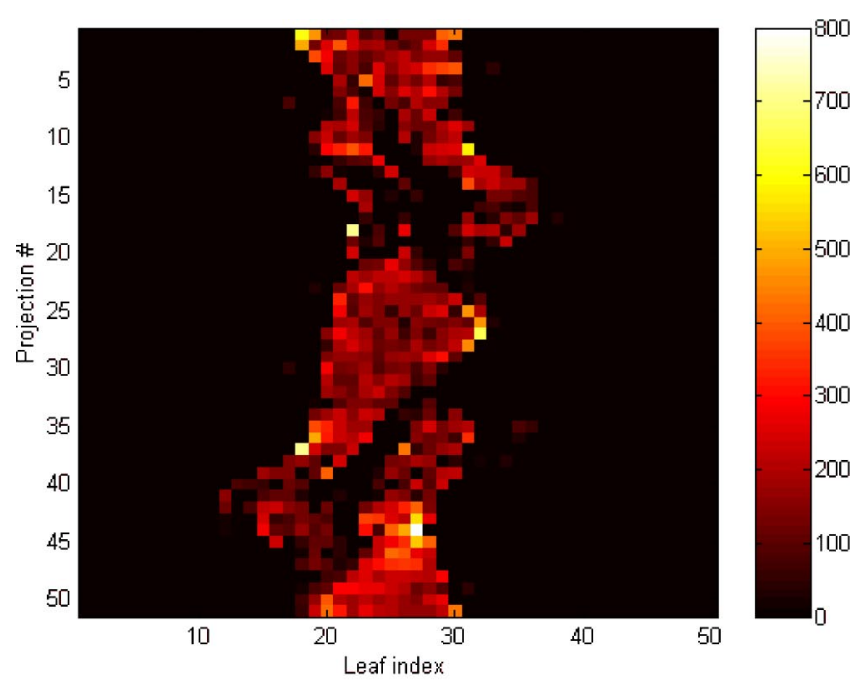

(a)

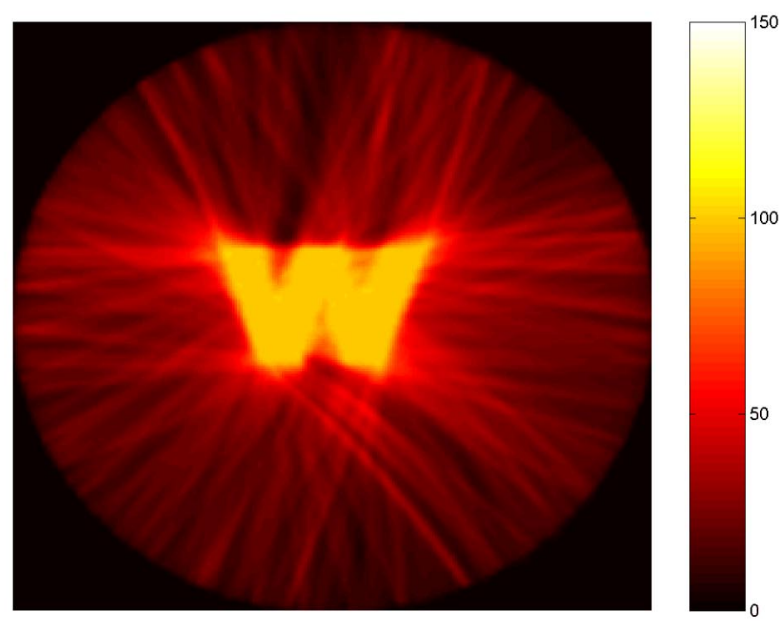

(b)

FIG. 9. Motion incorporated optimization results on a deforming target. (a) sinogram, (b) dose distribution. Dose was conformal to the "W" shape target. Similar dose conformality $(\mathrm{DSC}=0.932)$ and uniformity as in Fig. 7 were obtained. The prescribed dose was $95 \%$ to the target.

\section{E. Dice similarity coefficient conformality index}

A conformality index (CI) was used to evaluate treatment planning results. Usually the CI is simply defined as the ratio of the planning target volume (PTV) and the volume enclosed by prescription isodose surface. However the CI is not sensitive to target position change which often happens due to inter- and intra-fraction motion. Dice similarity coefficient $(\mathrm{DSC})^{21,22}$ is appropriate in comparison-of-agreement studies and has been used in image registration validation. It can also be a useful tool for dose conformality evaluation. The DSC conformality index is given by

$$
\mathrm{DSC}=\frac{2 a}{2 a+b+c}=\frac{2 n\left\{A_{1} \cap A_{2}\right\}}{n\left\{A_{1}\right\}+n\left\{A_{2}\right\}}
$$

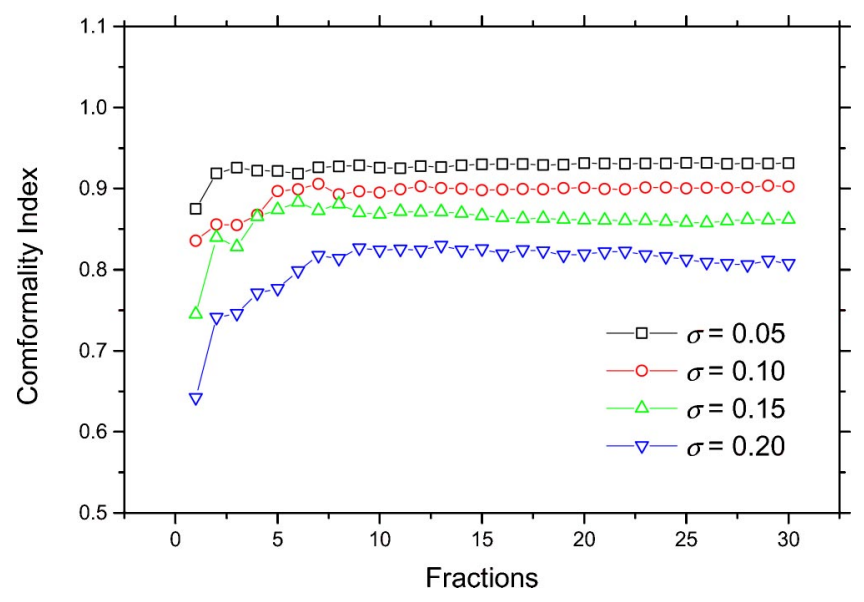

FIG. 10. DSC conformality indexes change with treatment fractions for tracking errors $\delta$ with different standard deviations of $\sigma$. Conformabilities were improved after a few fractions and then approached fixed values.

where $A_{1}$ is the set of voxels of PTV; $A_{2}$ is the set of voxels of the volume enclosed by $95 \%$ or other specified isodose surface; $a$, representing proper delivery, is the ratio of the number of voxels common to both data sets to the number of voxels of $A_{1} ; b$, representing underdose in the target, is the ratio of the number of voxels unique to $A_{1}$ to the number of voxels of $A_{1} ; c$, representing overdose in the normal tissue, is the ratio of the number of voxels unique to $A_{2}$ to the number of voxels of $A_{1}$. The DSC conformality index only goes to 1 when $95 \%$ or other prescription dose covers the PTV and no surrounding tissue receives dose more than that. The DSC conformality index was used to evaluate fractionation deliveries with tracking error.

\section{RESULTS}

\section{A. Phantom case}

With a given displacement vector field of the phantom shown in Fig. 3, a dynamic image sequence at different deformation phases was created by image warping according to Eq. (1b). The result of images at selected phases is shown in Fig. 5. The density changes are due to the deformation of the phantom.

The beamlet delivery phases are represented by the gantry angles when the beamlets are being delivered. With the preset phase correlation, beamlets were calculated using the images at the corresponding deformation phases. The deformation-free phase was chosen as the primary phase. Beamlets at other phases were then mapped back to the primary phase using the displacement vector fields. The shape of the beamlet was deformed after dose mapping due to the deformation of the phantom. A sample beamlet dose distribution before and after dose mapping is shown in Fig. 6 . With a prescribed dose of $95 \%$ to the target, conventional optimization according to Eq. (4a) was first performed on a stationary target. The optimized static plan is plotted as a sinogram shown in Fig. 7. The final dose distribution was computed using the static plan and beamlets calculated on the stationary phantom. The result is also shown in Fig. 7. 
TABLE I. DSC factors changes with fractions. Properly delivered portion $a$ was improved and overdose to normal tissue $c$ was decreased. However the underdose in the target $b$ increased too.

\begin{tabular}{crrrr}
\hline \hline \multicolumn{5}{c}{1 fraction } \\
\hline Error $\sigma$ & 0.05 & 0.10 & 0.15 & 0.20 \\
$a$ & 0.948 & 0.782 & 0.669 & 0.605 \\
$b$ & 0.125 & 0.102 & 0.099 & 0.106 \\
$c$ & 0.052 & 0.218 & 0.331 & 0.395 \\
\multicolumn{5}{c}{30 fractions } \\
Error $\sigma$ & 0.05 & 0.10 & 0.15 & \\
$a$ & 0.973 & 0.917 & 0.842 & 0.20 \\
$b$ & 0.127 & 0.129 & 0.124 & 0.141 \\
$c$ & 0.027 & 0.083 & 0.158 & 0.228 \\
\hline \hline
\end{tabular}

High dose conformality ( $\mathrm{DSC}=0.925)$ and uniformity were obtained. The "W" shape was clearly distinguishable from the dose distribution images.

To simulate the phantom under deformation with no motion mitigation applied, the delivery result was computed using the same plan [Fig. 7(a)] but with the dose mapped (deformed) beamlets [Fig. 6(b)]. The dose distribution was highly distorted as shown in Fig. 8. Severe hot and cold spots were observed. Dose conformality and uniformity were extremely impaired due to the target motion. DSC decreased from 0.925 to 0.590 due to the target motion.

With the same dose prescription, motion incorporated optimization gave an optimal plan for a target that deformed according to the preset delivery-deformation correlation. The optimized plan is plotted as a sinogram and shown in Fig. 9. The final dose distribution was reconstructed using the motion incorporated plan and the deformed beamlets. The result is also shown in Fig. 9. The dose was again highly conformal to the target and similar conformality ( $\mathrm{DSC}=0.932)$ and uniformity were observed on the dynamic case as the static case.

The synchronized delivery with human performance errors was studied according to Eq. (5b). Figure 10 shows the conformality index changing with treatment fractions for the tracking errors $\delta$ with different value of standard deviation $\sigma$. The tracking errors $\delta$ were generated by a random number generator implemented in MATLAB. DSC conformality index approached a fixed value after three to ten treatment fractions depending on the degree of tracking errors. Table I shows the

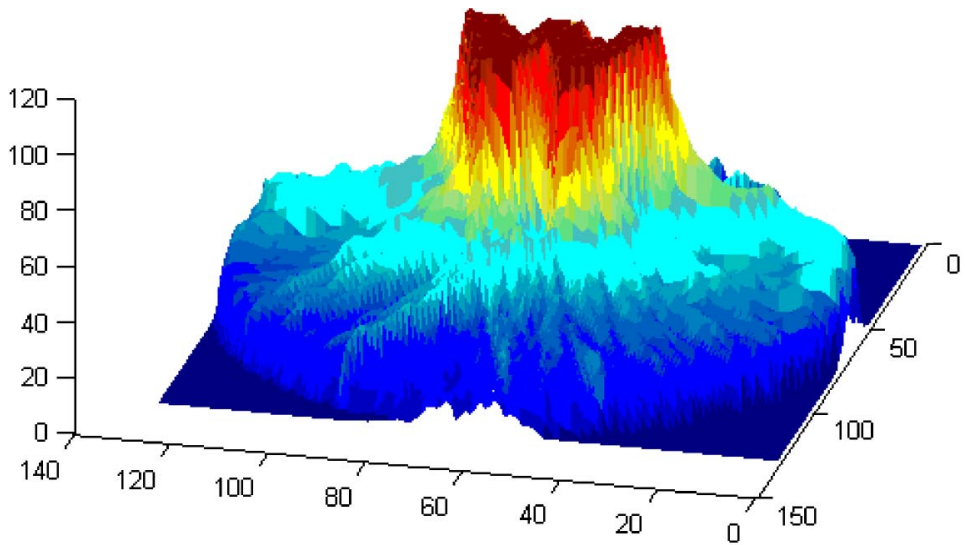

(a)

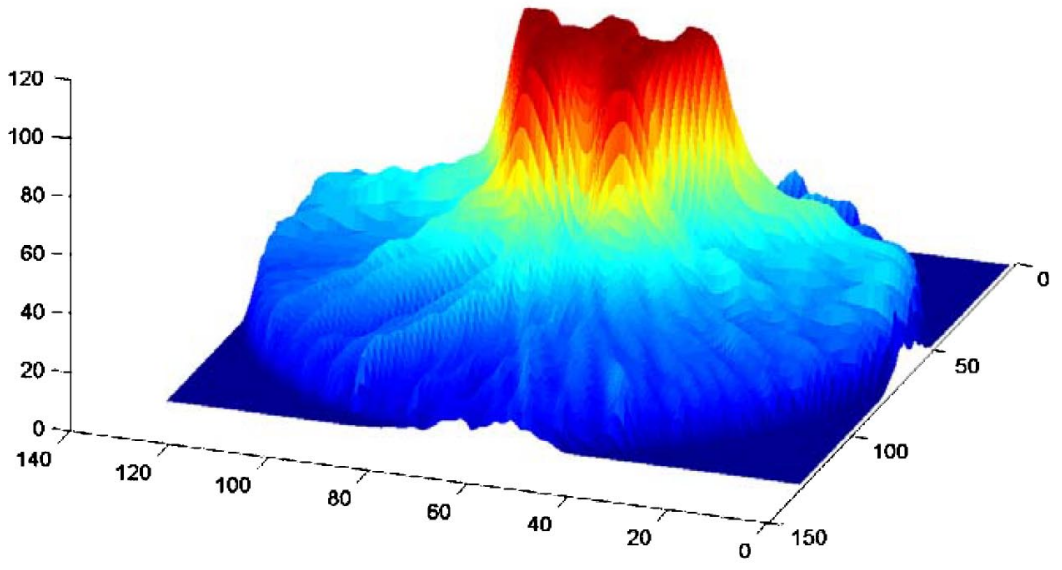

(b)
FIG. 11. Dose distributions after (a) treatment planning and (b) 30 deliveries. Tracking errors with standard deviation $\sigma$ of 0.1 were used in multi-fraction delivery simulation. 


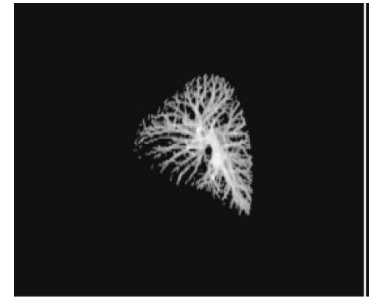

phase 1

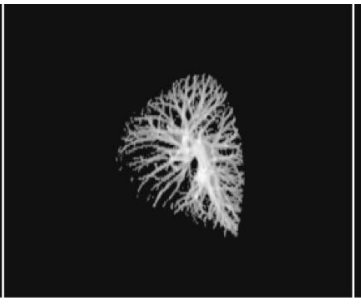

phase 2

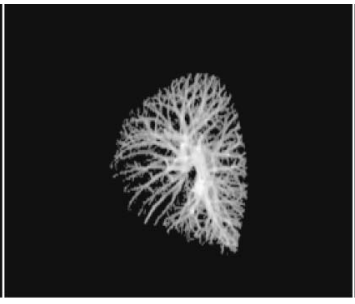

phase 3

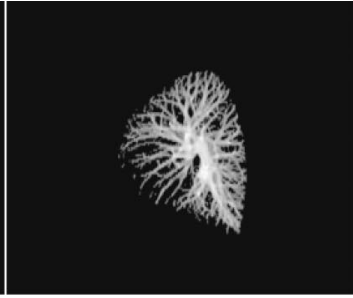

phase 4

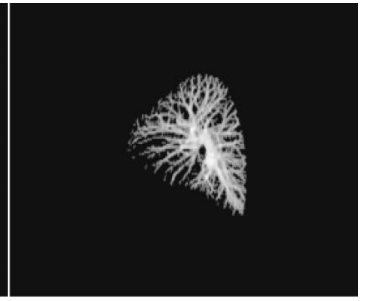

phase 5

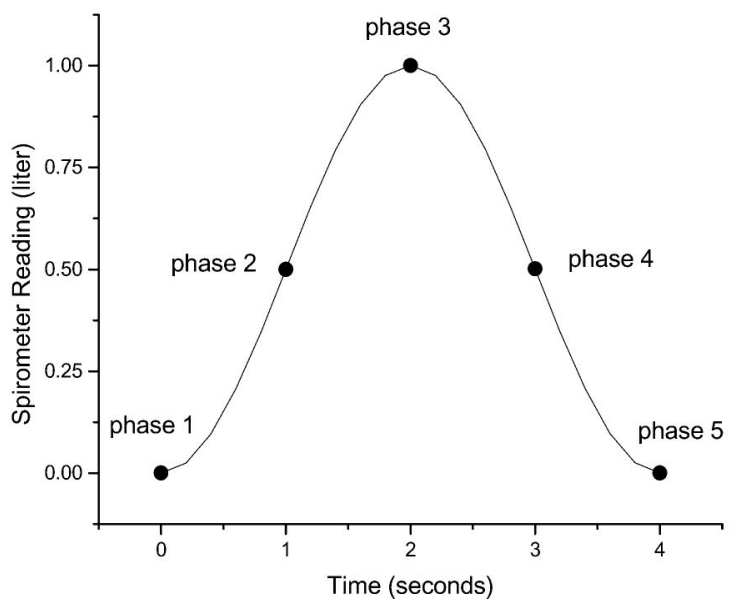

FIG. 12. Lung images at different breathing phases obtained by image warping and their positions in spirometer breathing signal.

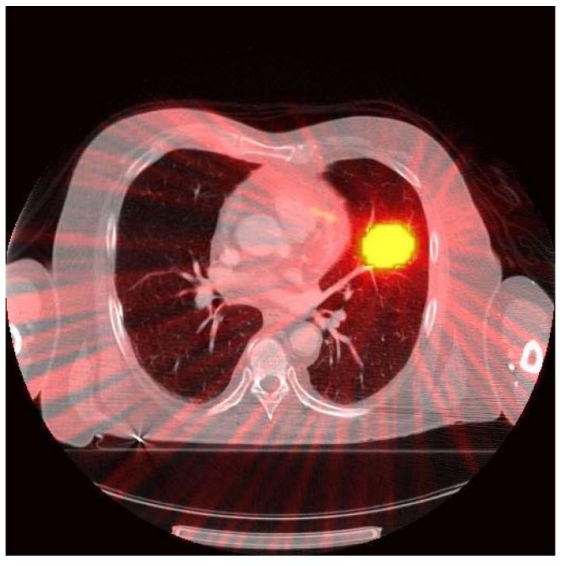

(a)

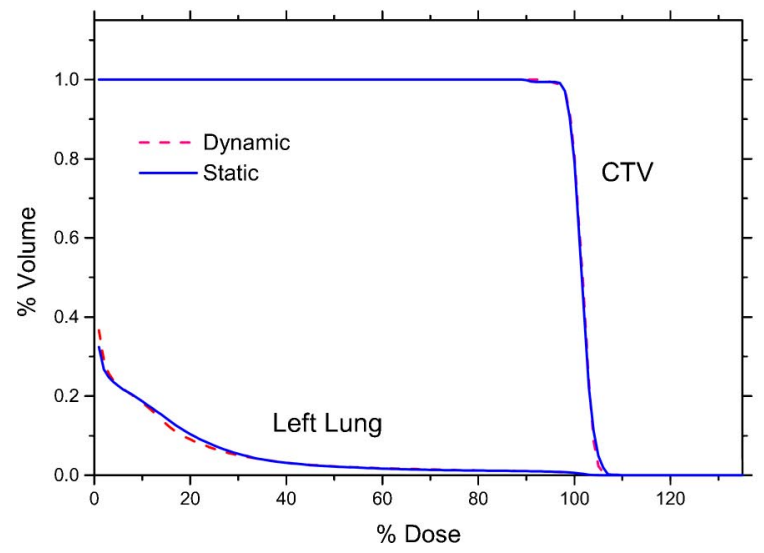

FIG. 13. (a) Dose distribution of treatment planning optimization incorporating motion. (b) DVH curves of the treatment plannings for dynamic delivery with synchronized breathing and conventional static delivery. $a, b, c$ values after one and thirty deliveries. Results show that the properly irradiated region in PTV was improved and overdose to surrounding tissue decreased after multiple deliveries. However underdose in PTV increased as well due to the decrease of dose gradients. Figure 11 shows the dose profiles of treatment planning and the result after 30 delivery fractions. The residual motion of multifraction delivery smoothed the dose distribution. However, with small residual motion, the dose was still conformal to the target. The "W" shape was clearly distinguishable in the dose profile.

\section{B. Human case}

A similar study was performed on a real human case. With the displacement maps shown in Fig. 4, a dynamic lung image sequence was created. Figure 12 shows the images at a few selected phases and their position in the breathing curve. The positions in the breathing curve were determined by calculating lung volume changes. ${ }^{18}$

A breathing cycle was selected from the patient's own free breathing curve as the guiding cycle. The breathing guide was obtained by repeating the guiding cycle. The delivery phases represented by projection number were then correlated to the breathing guide. With the delivery-breathing phase correlation, a motion incorporated optimization was performed on the 4D lung images. For comparison, another conventional optimization was performed on the static CT images. Figure 13 shows the treatment planning results. In both cases, the CTV was nicely covered. The dose was highly conformal to the target.

The breathing guide and breathing curve were displayed 


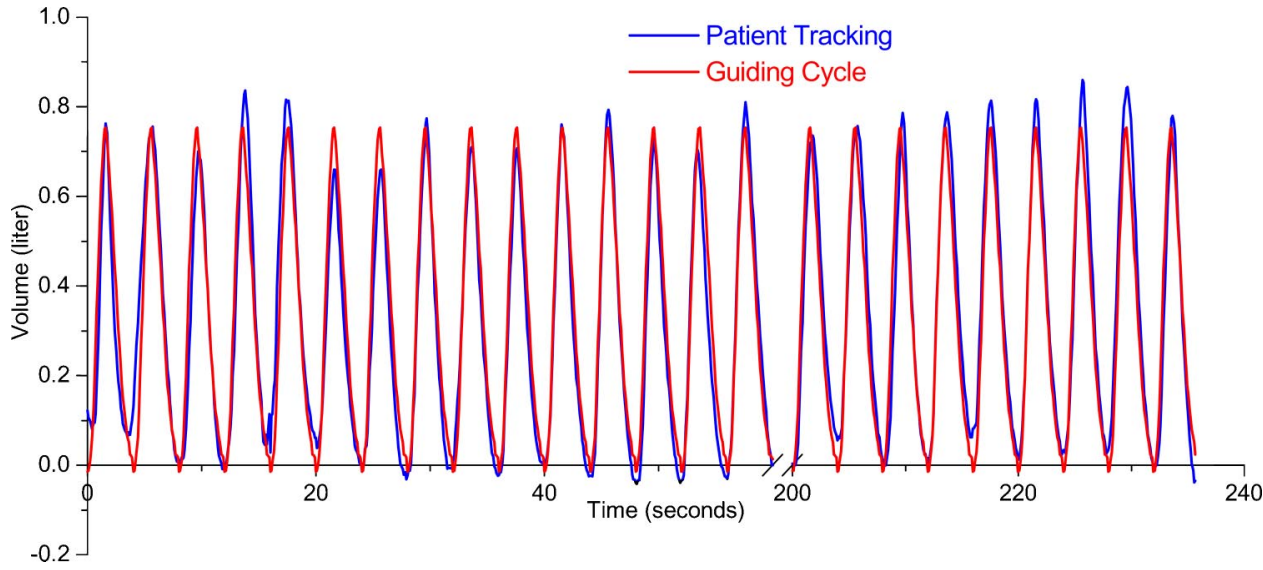

FIG. 14. Breathing tracking performance of a healthy volunteer. Guiding cycles and patient's breathing curve were displayed by video glasses. to the patient through video glasses. Figure 14 shows a healthy volunteer's ability to track his breathing guide. During the 4 min test, he was able to control his breath to track the guiding cycles with a certain accuracy. To a first-order approximation, the target motion may be considered proportional to the spirometer reading. The free breathing motion and residual motion of breathing tracking were plotted as histogram in Fig. 15. Breathing signal tracking errors have normal distribution with a standard deviation of 0.0661 . Normalized by the amplitude of breathing curve, the relative tracking errors $\delta$ in Eq. (5b) had a standard deviation $\sigma$ of 0.082 .

To simulate fractioned deliveries, the actual tracking errors from the breathing curve in Fig. 14 were used for the $\delta$

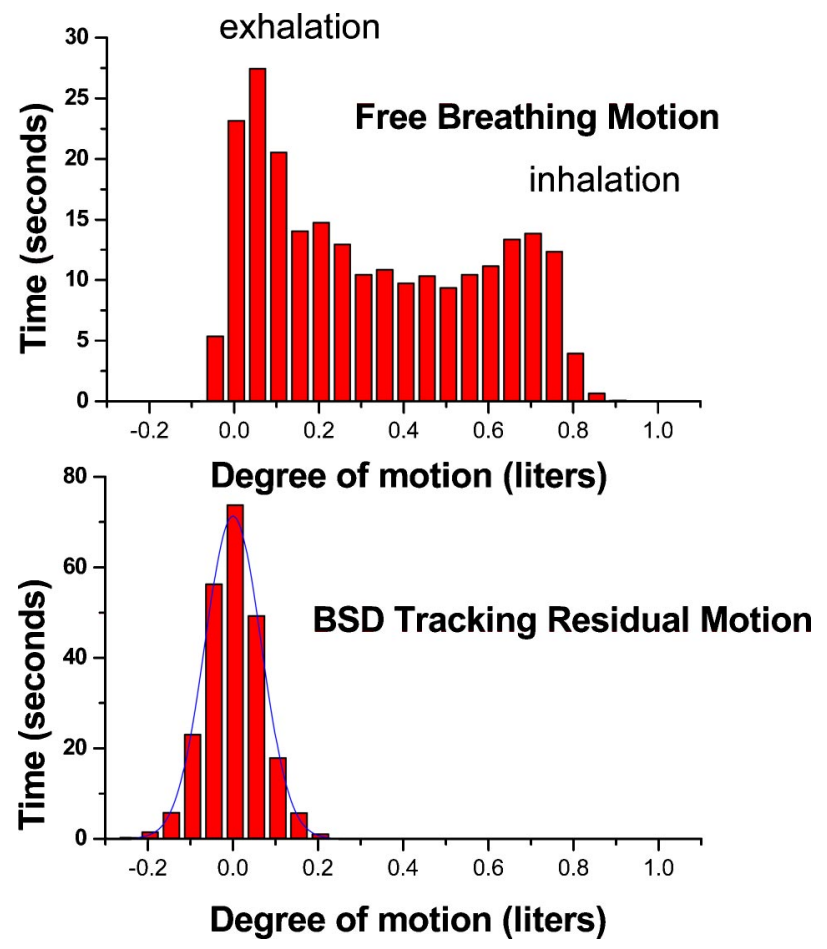

FIG. 15. Motion reduction by breathing tracking. The breathing signal tracking errors have a normal distribution with a standard deviation of 0.066 liter. Normalized to the amplitude of free breathing, the relative standard deviation $\sigma$, a measure of tracking error $\delta$, is about 0.082 . in Eq. (5b). The starting points were selected randomly for each fraction. Figure 16 shows the dose histogram for treatment planning, 1, 15, and 30 fractions.

\section{DISCUSSION}

Helical tomotheray is able to deliver highly conformal dose to the target with a properly optimized plan. However, without any motion mitigation method, the actual dose delivered may be significantly different from what is predicted by treatment planning. This study presents a new motion compensation method for treatment plan optimization and delivery. Different from other motion control techniques whose dose calculations are based on static CT images, this new method performs treatment planning optimization on a dynamic image sequence.

In this study, dynamic image sequence was obtained by image warping. In clinical practice, a 4D image sequence obtained by dynamic CT scans would be performed. ${ }^{23,24}$ Hysteresis in the target trajectory was observed in some patients due to the different mechanism between inspiration and expiration. ${ }^{25}$ The 4D images from breath holding CT scans and image warping do not model hysteresis information.

The key to this new method is synchronized delivery. The correlation between the treatment delivery and breathing cycle is set in the treatment planning. The correlation should be preserved during treatment delivery. This is achieved by instructing the patient to breathe following guiding cycles. Preliminary trials on healthy volunteers show that tracking the guiding cycle is much easier than breath holding. The tracking errors have a normal distribution and the residual motion is much less than the motion of free breathing. Future clinical trials on lung cancer patients are under way.

In contrast to conventional $\mathrm{CI}$, which is often used to evaluate treatment planning results, the DSC conformality index is more sensitive to motion and setup error and thereby is more suitable for delivery error evaluation. Computer simulated tracking error studies showed conformality decreases as tracking error increases. Conformability was improved after several delivery fractions and then converged to a fixed value. After 30 delivery fractions, underdose to the target increased and overdose to normal tissue decreased as 


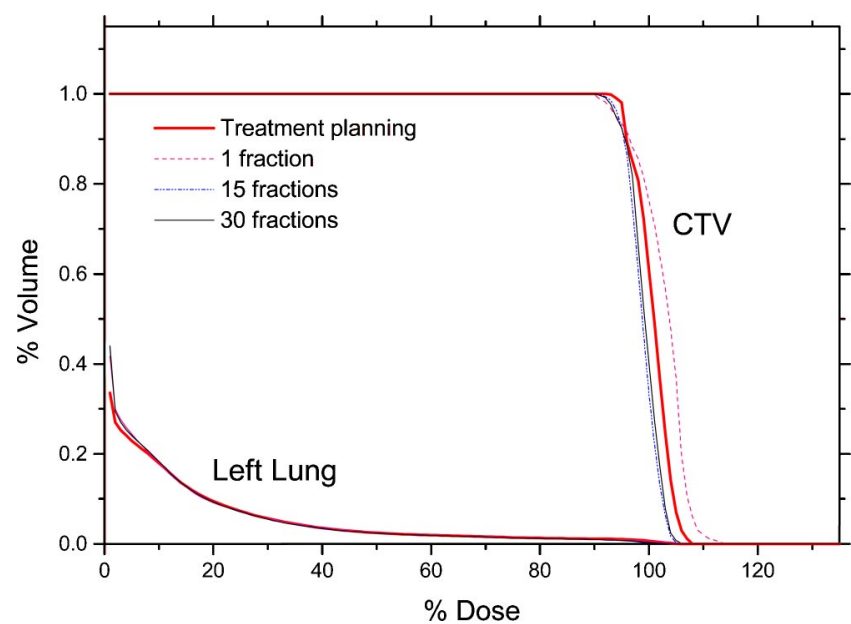

FIG. 16. DVH curve changes with fractioned deliveries. Human tracking errors were included in dose calculation.

dose gradient decreased due to motion blurring. The tracking residual motion blurring was much smaller than that of free breathing motion. As a consequence, the final dose distribution after multiple fraction deliveries preserved most of the high dose gradients.

However, if the beamlets last for more than one half breathing period, they are totally blurred by the motion and the dose gradient will not be improved. Tomotherapy gantry rotates with a period of $20 \mathrm{~s}$ and there are total of 51 projections in each rotation. So each beamlet lasts less than $0.4 \mathrm{~s}$ depending on its intensity. Therefore, beamlet blurring from respiratory motion would be negligible. Cardiac motion is much faster than respiratory motion and most beamlets last for more than one half cardiac cycle. As a consequence, this method will not be able to compensate for cardiac motion. Fortunately for most parts of the lung, cardiac motion is much smaller than respiratory motion in magnitude.

This new method delivers radiation continuously and does not prolong treatment time. Assuming linear translation target motion, another continuous delivery method-the "tracking" technique-may oversimplify target motion. Also superimposing target motion on leaf motion may cause delivery difficulties due to MLCs mechanical limitations. This new method, in principle, only imposes an assumption of reproducible target motion and is not constrained by dynamic MLC mechanical limitation.

For beamlet-based inverse treatment planning, the most computationally expensive procedure is the beamlet calculation. In this study, in order to utilize the commercial optimizer, five sets of beamlets were calculated for 10 breathing phases. In the future, beamlet calculation may be guided to select the CT image at a specific phase from a 4D image sequence according to the delivery-deformation phase correlation. Approximately the same number of beamlets are needed for optimization. Thus no extra computational cost is imposed on the beamlet calculations. Extra computational cost is needed for the three-dimensional deformable image registration and the dose mapping. However, the CPU time needed by these processes is negligible when compared with that of beamlet calculation.

This new method is especially suitable for tomotherapy since the delivery-deformation correlation is determined prior to treatment optimization. For conventional IMRT delivery using dynamic MLC, this correlation is not known until the leaf sequence is calculated by the sequencer from the optimization result. The problem could be solved by iterative motion incorporated optimization as shown in Fig. 17. At the beginning, optimization is performed using the primary phase $\mathrm{CT}$ images assuming no motion. The deliverydeformation phase correlation can then be determined from the leaf sequence of the optimization result. Beamlets are deformed according to this correlation. A motion incorporated optimization is performed. This process repeats until the newly generated intensity map from optimization is very close to the one from the last loop. However, the convergence and outcomes of this iterative optimization require testing.

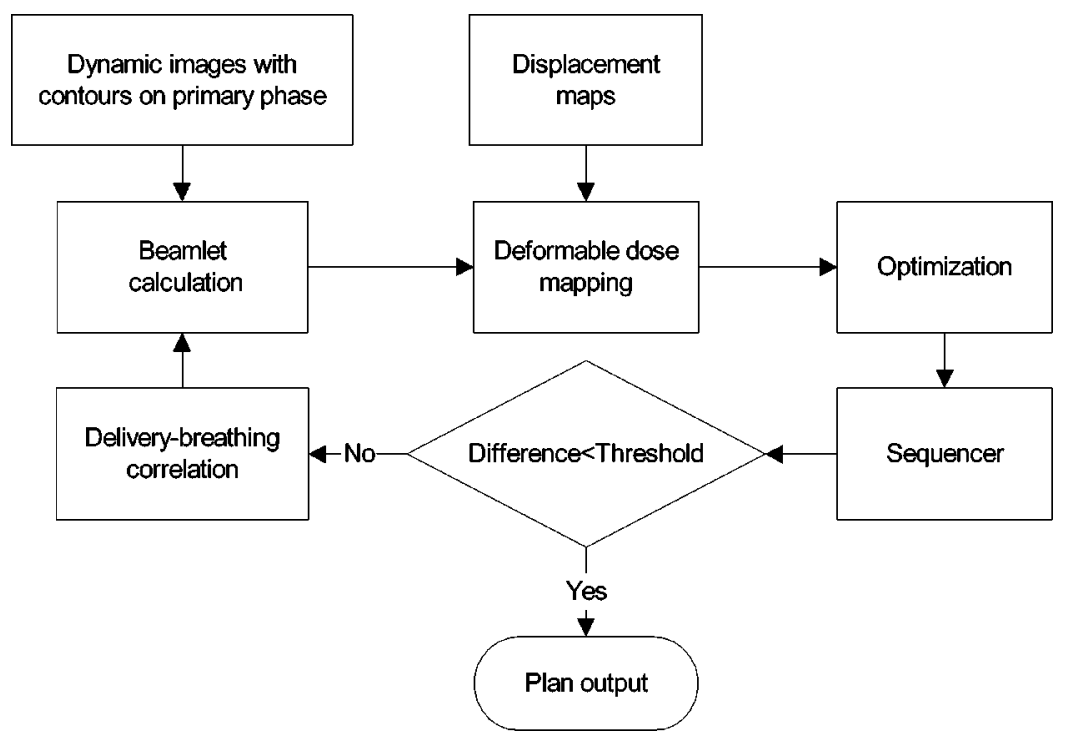

FIG. 17. Scheme of the optimization incorporating motion for conventional IMRT delivery using a dynamic MLC. 


\section{CONCLUSION}

With the knowledge of the deformation state at each delivery phase, target motion/deformation can be incorporated into the treatment plan optimization. In principle, this method does not impose any limitation on target motion other than periodic reproducibility and may provide an accurate dose prediction in treatment planning. Motion incorporated optimization on a mobile target results in highly conformal dose distribution. This method is especially suitable for tomotherapy whose delivery-breathing phase correlation is determined before optimization. This method may also be possible to apply to conventional IMRT delivery using iterative optimization with extra computational cost.

\section{ACKNOWLEDGMENTS}

This work was supported by a grant from the National Institute of Health (Award No. P01 CA-88960). The authors also want to thank Peter Hoban Ph.D. of Siemens Medical System for providing superposition/convolution code for dose calculation.

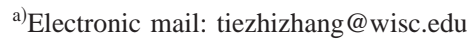

${ }^{1}$ T. R. Mackie, T. Holmes, S. Swerdloff, P. Reckwerdt, J. O. Deasy, J. Yang, B. Paliwal, and T. Kinsella, "Tomotherapy: A new concept for the delivery of dynamic conformal radiotherapy," Med. Phys. 20, 1709-1719 (1993).

${ }^{2}$ J. S. Welsh, R. R. Patel, M. A. Ritter, P. M. Harari, T. R. Mackie, and M. P. Mehta, "Helical tomotherapy: An innovative technology and approach to radiation therapy," Technol. Cancer Res. Treat. 1, 311-316 (2002).

${ }^{3}$ R. A. Scrimger, W. A. Tome WA, G. H. Olivera, P. J. Reckwerdt, M. P. Mehta, and J. F. Fowler, "Reduction in radiation dose to lung and other normal tissues using helical tomotherapy to treat lung cancer, in comparison to conventional field arrangements," Am. J. Clin. Oncol. 26, 70-78 (2003)

${ }^{4}$ S. Shimizu, H. Shirato, S. Ogura, H. Akita-dosaka, K. Kitamura, T. Nishioka, K. Kagei, M. Nishimura, and K. Miyasaka, "Detection of lung tumor movement in real-time tumor-tracking radiotherapy," Int. J. Radiat. Oncol., Biol., Phys. 51, 304-310 (2001).

${ }^{5}$ J. N. Yang, T. R. Mackie, P. Reckwerdt, J. O. Deasy, and B. R. Thomadsen, "An investigation of tomotherapy beam delivery," Med. Phys. 24, 425-436 (1997).

${ }^{6}$ C. X. Yu, D. A. Jaffray, and J. W. Wong, "The effects of intra-fraction organ motion on the delivery of dynamic intensity modulation," Phys. Med. Biol. 43, 91-104 (1998).

${ }^{7}$ T. Bortfeld, K. Jokivarsi, M. Goitein, J. Kung, and S. Jiang, "Effects of intra-fraction motion on IMRT dose delivery: Statistical analysis and simulation," Phys. Med. Biol. 47, 2203-2219 (2002).

${ }^{8}$ S. B. Jiang, C. Pope, K. M. Al Jarrah, J. H. Kung, T. Bortfeld, and G. T. Y. Chen, "An experimental investigation on intra-fractional organ motion effects in lung IMRT treatments," Phys. Med. Biol. 48, 1773-1784 (2003).

${ }^{9}$ T. Tada, K. Minakuchi, T. Fujioka, M. Sakurai, M. Koda, I. Kawase, T. Nakajima, M. Nishioka, T. Tonai, and T. Kozuka, "Lung cancer: Inter- mittent irradiation synchronized with respiratory motion-results of a pilot study," Radiology 207, 779-783 (1998).

${ }^{10}$ H. D. Kubo, P. M. Len, S. Minohara, and H. Mostafavi, "Breathing synchronized radiotherapy program at the University of California Davis Cancer Center," Med. Phys. 27, 346-353 (2000).

${ }^{11}$ R. Wagman, E. Yorke, E. Ford, P. Giraud, G. Mageras, R. Minsky, and K. Rosenzweig, "Respiratory gating for liver tumors: Use in dose escalation,” Int. J. Radiat. Oncol., Biol., Phys. 55, 659-668 (2003).

${ }^{12}$ J. Hanley, M. M. Debois, D. Mah, G. S. Mageras, A. Raben, K. Rosenzweig, B. Mychalczak, L. H. Schwartz, P. J. Gloeggler, M. T. (A.S.C.P.), W. Lutz, C. C. Ling, S. A. Leibel, Z. Fuks, and G. J. Kutcher, “Deep inspiration breath-hold technique for lung tumors: The potential value of target immobilization and reduced lung density in dose escalation," Int. J. Radiat. Oncol., Biol., Phys. 45, 603-611 (1999).

${ }^{13}$ J. W. Wong, M. B. Sharpe, D. A. Jaffray, V. R. Kini, J. M. Robertson, J. S. Stromberg, and A. A. Martinez, "The use of active breathing control (ABC) to reduce margin for breathing motion," Int. J. Radiat. Oncol., Biol., Phys. 44, 911-919 (1999).

${ }^{14}$ L. A. Dawson, K. K. Brock, S. Kazanjian, D. Fitch, C. J. McGinn, T. S. Lawrence, R. K. Ten Haken, and J. Balter, "The reproducibility of organ position using active breathing control (ABC) during liver radiotherapy," Int. J. Radiat. Oncol., Biol., Phys. 51, 1410-1421 (2001).

${ }^{15}$ T. Neicu, H. Shirato, U. Seppenwoolde, and S. Jiang, "Synchronized moving aperture radiation therapy (SMART): Average tumour trajectory for lung patients," Phys. Med. Biol. 48, 587-598 (2003).

${ }^{16}$ P. J. Keall, V. R. Kini, S. S. Vedam, and R. Mohan, "Motion adaptive X-ray therapy: A feasibility study,' Phys. Med. Biol. 46, 1-10 (2001).

${ }^{17}$ K. M. Brock, J. M. Balter, L. A. Bawson, M. L. Kessler, and C. R. Meyer, "Automated generation of a four-dimensional model of the liver using warping and mutual information," Med. Phys. 30, 1128-1133 (2003).

${ }^{18}$ T. Zhang, H. Keller, S. Fain, T. R. Mackie, and B. Paliwal, "Three dimensional lung respiratory motion study using finite element analysis," Med. Phys. 30, 1346 (2003).

${ }^{19}$ T. Zhang, T. R. Mackie, and B. Paliwal, "Technical note: Deformable image registration of the lung using finite element contact-impact analysis," Med. Phys. (submitted).

${ }^{20}$ T. Zhang, H. Keller, M. O’Brien, T. R. Mackie, and B. Paliwal, “Application of the spirometer in respiratory gated radiotherapy," Med. Phys. 30, 3165-3172 (2003).

${ }^{21}$ J. L. Fleiss, "Measures of the amount of ecologic association between species," Ecology 26, 297-302 (1945).

${ }^{22}$ A. Bharatha, M. Hirose, N. Hata, S. K. Warfield, M. Ferrant, K. H. Zou, E. Suarez-santana, J. Ruiz-Alzola, A. D'Amico, R. A. Cormack, R. Kikinis, F. A. Jolesz, and C. M. C. Tempany, "Evaluation of threedimensional finite element-based deformable registration of per- and intraoperative prostate imaging," Med. Phys. 28, 2551-2560 (2001).

${ }^{23}$ S. S. Vedam, P. J. Keall, V. R. Kini, H. Mostafavi, and H. P. Shukla, "Acquiring a four-dimensional computed tomography dataset using an external respiratory signal," Phys. Med. Biol. 48, 45-62 (2003).

${ }^{24}$ D. A. Low, M. Nystrom, E. Kalinin, P. Parikh, J. F. Dempsey, J. D. Bradley, S. Mutic, S. H. Wahab, T. Islam, G. Christensen, D. G. Politte, and B. R. Whiting, "A method for the reconstruction of four-dimensional synchronized CT scans acquired during free breathing," Med. Phys. 30, 1254-1263 (2003).

${ }^{25}$ Y. Seppenwoolde, H. Shirato, K. Kitamura, S. Shimizu, M. Van Herk, J. V. Lebesque, and K. Miyasaka, "Precise and real-time measurement of 3D tumor motion in lung due to breathing and heatbeat, measured during radiotherapy," Int. J. Radiat. Oncol., Biol., Phys. 53, 822-833 (2002). 